\title{
Utilisation des techniques d'imagerie pour la cartographie des vitesses à la surface d'une avalanche dense
}

\author{
F. Granada \\ Laboratoire d'Instrumentation de Micro-informatique et d'électronique - CEMAGREF
}

\author{
O. Marco
}

Division Nivologie du CEMAGREF

P. Villemain

Laboratoire d'Instrumentation de Micro-informatique et d'électronique

\begin{abstract}
L'étude de la dynamique des avalanches a donné lieu à de nombreux modèles théoriques et à quelques expérimentations sur des sites avalancheux pour les valider. Nous proposons, dans cet article, une méthode d'analyse de films vidéo d'avalanches de neige dense avec pour objectif de réaliser une cartographie des vitesses à la surface de l'écoulement et de comparer ces résultats à ceux des modèles numériques. Les conditions des prises de vues sont très mal maîtrisées en raison du danger que représente l'avalanche pour l'opérateur. Le matériel de prise de vue est standard, car il ne peut être question d'utiliser des caméras à haute résolution ou à grande vitesse qui, par leur poids et leur sensibilité à la température, sont inadaptées. Les traitements doivent pouvoir donner des résultats sur des images extraites des documents vidéo ou films, obtenues par un matériel standard. Dans ces conditions, les images obtenues présentent deux difficultés pour leur traitement : la fréquence de 25 images/s conduit à un « flou de bougé " dû au temps de pose et, d'autre part, les formes et contrastes des images sont mal définis et instables au cours du temps. Cependant, des études ont été conduites avec succès sur des phénomènes semblables en météorologie ou en aérodynamique. L'analyse par autocorrélation et intercorrélation fournit des résultats corrects. Deux approches sont présentées ici. La première utilise l'intercorrélation entre images successives et a permis la représentation de l'évolution de la vitesse d'une portion de surface au cours du temps en quelques points de la surface de l'écoulement. La seconde utilise une méthode de filtrage et a permis d'obtenir la répartition des vitesses en tout point de surface de l'écoulement. Les résultats obtenus sont bidimensionnels car nous avons exclu de cette étude le problème de la restitution tridimensionnel des images qui sera traité ultérieurement.
\end{abstract}

\section{Q MESURE DE LA VITESSE PAR INTER- CORRÉLATION D'IMAGES}

\subsection{Techniques [1]}

L'analyse des informations contenues sur une image est réalisée par un traitement numérique de signaux bidimensionnels. Nous pouvions traiter la mesure de la vitesse de l'avalanche à la surface de l'écoulement, en utilisant :

- les techniques issues de la topologie mathématique permettant la reconnaissance d'une forme à une autre, ou - les techniques du traitement du signal correspondant à une analyse quantitative des informations présentes sur l'image.

L'information sur le mouvement de la neige en surface est essentiellement due à la présence de boules dans le corps d'une avalanche dense et en particulier à la surface de l'écoulement. Une méthode basée sur la reconnaissance des formes peut être envisagée mais après avoir examiné attentivement les films, nous nous sommes aperçus que les boules sont peu différenciées, seule une petite portion est visible, rendant la surface de l'avalanche moutonnée. Une première estimation de la vitesse a été obtenue en pointant des boules de grand diamètre d'une image à l'autre. Mais le nombre de ces boules était trop faible pour permettre un traitement automatisé. Cette méthode fut donc utilisée manuellement et ponctuellement pour vérifier la précision des résultats obtenus avec la seconde méthode.

Les boules provoquent des zones d'ombre et donc des changements d'intensité lumineuse très nets et ponctuels ; la mesure du déplacement de ces zones d'ombre permet une première analyse de la vitesse. La fiabilité de cette 
méthode repose sur la faible variation de forme de cette zone d'ombre d'une image à une autre. L'information retenue est contenue dans l'intensité lumineuse de chaque pixel. Nous avons travaillé sur des images en noir et blanc.

Pour réaliser ce travail nous avons utilisé un logiciel de traitement d'images C-IMA pour PC AT développé par l'équipe du professeur Schon au Laboratoire de Traitement du Signal et d'Instrumentation à l'Université Jean Monnet de St-Etienne.

La numérisation des informations analogiques présentes sur une image est réalisée à l'aide d'une carte type Matrox (PIP 1024B). Après numérisation, l'image en noir et blanc obtenue est formée de $512 \times 512$ pixels, chacun étant affecté d'une valeur comprise entre 0 et 255 correspondant à son niveau de gris (le noir correspond à 0 et le blanc à 255). Le logiciel C-IMA permet alors de définir une fenêtre rectangulaire délimitant une petite surface de l'image étudiée. Il utilise ensuite les méthodes d'intercorrélation [2], [3], [4] pour comparer l'information contenue dans cette fenêtre à celle présente dans la même fenêtre mais placée sur une image différente. Le logiciel envisage alors tous les déplacements possibles de la première image et évalue, dans chaque cas, la corrélation existant entre les pixels des deux fenêtres en se basant sur le niveau de gris. Le logiciel présente alors les résultats sous la forme d'un corrélogramme bidimensionnel et indique le déplacement pour lequel cette intercorrélation est la maximale.

Mathématiquement, la méthode est la suivante :

Soit un repère orthonormé dont l'origine est le coin gauche supérieur de l'image. Soit $A$ l'ensemble de couples $(x, y)$ correspondant aux coordonnées des pixels contenus dans la fenêtre définie sur la première image et soient les fonctions $G 1(x, y)$ et $G 2(x, y)$, les fonctions intensités des images 1 et 2 . Le logiciel va alors étudier tous les déplacements possibles $(a, b)$ de l'ensemble des pixels contenus dans la fenêtre de la seconde image et faire correspondre, à chaque déplacement, la valeur $\operatorname{Cor}(a, b)$ de la fonction d'intercorrélation suivante :

$$
\operatorname{Cor}(a, b)=\sum \sum_{A} G_{1}(x, y) G_{2}(x+a, y+b)
$$

Pour représenter ces résultats sur un corrélogramme bidimensionnel [5], [6], le logiciel affecte d'une manière linéaire un niveau de gris à chaque couple $(a, b)$ selon la valeur de l'intercorrélation correspondant à ce point, en donnant une valeur nulle (noir) au couple pour lequel la fonction Cor est minimale et une valeur égale à 255 (blanc) au couple pour lequel cette fonction est maximale. On obtient alors le corrélogramme de la figure 1 .

La distance de déplacement, en pixels, est obtenue en calculant la distance entre le centre du corrélogramme et le pixel correspondant à la valeur maximale de la fonction d'intercorrélation. Nous pouvons situer visuellement ce point qui est au centre de la zone la plus claire du corrélogramme. Ce résultat est donné en pixels par le logiciel dans les deux directions $o x$ et $o y$. Il correspond au déplacement moyen de l'ensemble des points situés dans la fenêtre initiale.

\subsection{Présentation du site expérimental}

Il est situé au Col du Lautaret (Hautes Alpes) sur des pentes exposées au Sud-Est et dominées par la crête de Chaillol. Les altitudes de départ des avalanches sont comprises entre 2300 et $2600 \mathrm{~m}$ et les zones d'arrêt se trouvent vers $2100 \mathrm{~m}$.

Ce site est utilisé depuis 1972. Il a été équipé à l'origine par la Division Nivologie du CTGREF (devenu CEMAGREF) avec la collaboration du Laboratoire d'Applications Spéciales de la Physique du Centre d'Etudes Nucléaires de Grenoble (CENG).

Entre 1972 et 1981 de nombreuses expériences ont été réalisées sur ce site [1].

En 1989, un support de capteurs profilé en aile d'avion a été installé dans un couloir (voir fig. 3). En 1993, une plate-forme a été installée en rive gauche de ce même couloir pour permettre la réalisation de films vidéo.

\subsection{Résultats obtenus par intercorrélation}

L'avalanche que nous avons analysée a été déclenchée artificiellement le 7 janvier 1992 vers 14 heures dans ce couloir du site expérimental. Ses principales caractéristiques sont :

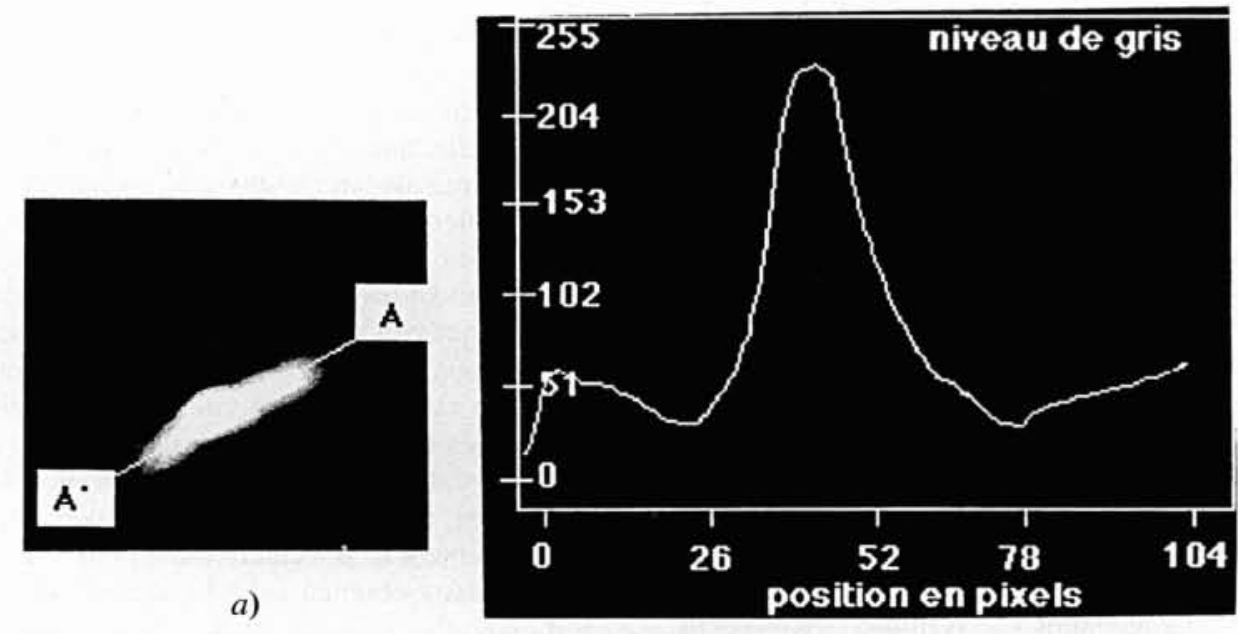

b)

1. Copie d'écran d'un corrélogramme obtenu entre deux images en utilisant le logiciel C-IMA.

a) Intercorrélogramme.

b) Coupe de cet intercorrélogramme selon l'axe $A A^{\prime}$ '. 
- Altitude de départ : $2425 \mathrm{~m}$.

- Fracture : une dizaine de centimètres d'épaisseur pour une longueur de $30 \mathrm{~m}$ environ.

- Altitude d'arrivée : $2221 \mathrm{~m}$.

- Vitesse du front au niveau du support de capteurs: $7,3 \mathrm{~m} / \mathrm{s}$.

- Durée de passage au niveau du support de capteurs : $10 \mathrm{~s}$.

- Neigè en place composée de grains fins ayant une masse volumique de $320 \mathrm{~kg} / \mathrm{m}^{3}$ environ pour une profondeur de $0,6 \mathrm{~m}$. Cette couche était humidifiée sur une dizaine de centimètres. Cette avalanche est une coulée superficielle.

La dimension de l'écoulement n'a pas permis d'analyser la vitesse en plusieurs points. Les résultats après traitement informatique et restitution des déplacements obtenus dans les images vers l'espace objet présentés à la figure 2 correspondent à la vitesse au milieu de la surface de l'écoulement.

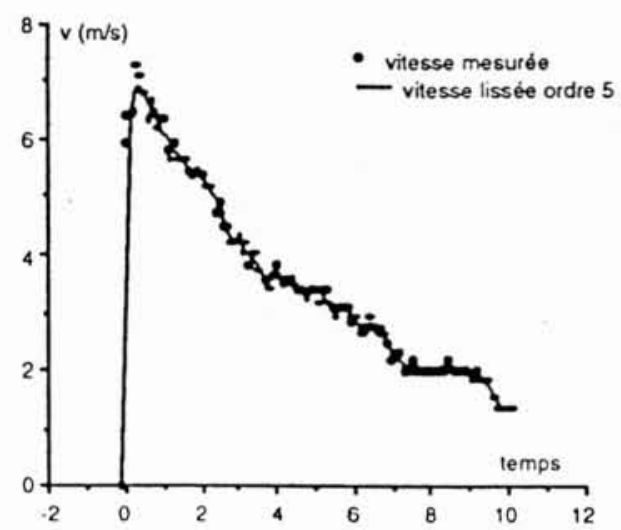

2. Vitesse à la surface de l'avalanche du 7 janvier 1992.

L'intérêt de ce profil est qu'il permet de retrouver le profil théorique d'une avalanche en phase d'arrêt [7]. Selon cet auteur, une avalanche dense est caractérisée par un front, un corps, qui a une vitesse identique à celle du front, et enfin une queue dont la vitesse décroît pratiquement linéairement. Une avalanche dense peut s'arrêter sur une pente, seulement à partir du moment où le corps de l'écoulement disparaît. Pendant cette phase d'arrêt, l'avalanche n'est composée que d'un front et d'une queue. Nos mesures traduisent très bien cette interprétation physique sachant que la coulée s'est arrêtée seulement 15 mètres à l'aval de notre point de mesure.

L'estimation des erreurs des mesures est très délicate. Toutefois les résultats obtenus sont du bon ordre de grandeur; les vérifications réalisées manuellement à l'écran (suivi d'une boule particulière entre deux points particuliers de l'image) concordent avec ces valeurs à $1,5 \mathrm{~m} / \mathrm{s}$ près.

\subsection{Limites de cette méthode}

La précision obtenue reste tout à fait insuffisante pour la réalisation d'une cartographie de la vitesse. Cette imprécision est due essentiellement à une erreur d'estimation du maximum du corrélogramme. Son application à des images présentant un "flou de bougé ", dû à des vitesses importantes de l'écoulement s'est révélée impossible. Nous avons donc été amenés à utiliser une méthode adaptative permettant l'élimination des informations relatives à des phénomènes autres que le simple déplacement.

\section{MÉTHODE ADAPTATIVE}

\subsection{Analyse du problème}

La technique que nous développons maintenant utilise le fait que chaque image vidéo est constituée par deux trames acquises à des instants séparés de 1/50 $\mathrm{s}$. Ceci nous permet de doubler la résolution temporelle de notre échantillonnage. D'autre part le "flou de bougé ", dû au temps de pose, produit dans toute méthode d'intercorrélation une contribution importante dans le pic central a pour effet de masquer le pic d'intercorrélation dû au déplacement. Pour remédier à cet inconvénient, nous avons simplement travaillé par soustraction de trames. Si l'on soustrait les trames paires et les trames impaires de deux images consécutives, nous obtenons deux images dont les niveaux de gris caractérisent les zones de la surface qui ont subi un déplacement. Nous n'avons ainsi retenu dans chaque image que les informations relatives aux déplacements (fig. 3). Il faut alors estimer la translation qui fait passer de la différence des trames paires à la différence des trames impaires. Nous utilisons pour cette estimation un filtre adaptatif de Wiener, déjà largement décrit dans de nombreux articles [8-13].

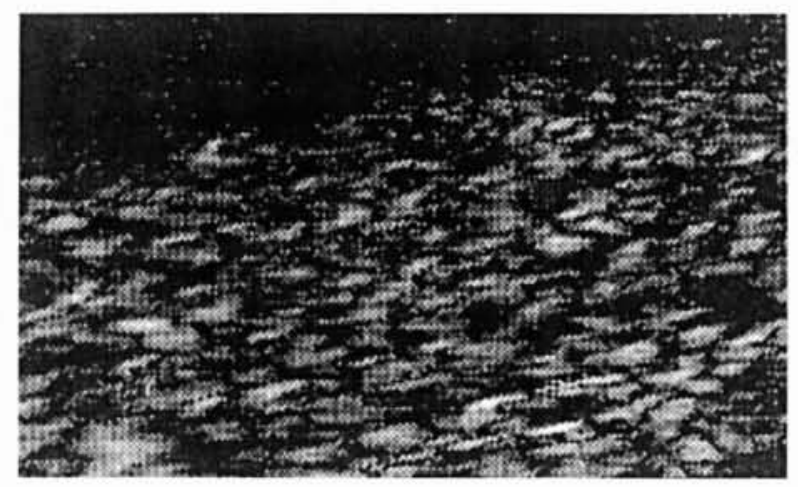

3. Soustraction de deux trames d'une même image.

\subsection{Modélisation}

On désigne par $J(x, y, t)$ l'intensité lumineuse de l'image initiale présentant le «flou de bougé » où $x, y$ sont les coordonnées d'un pixel de l'image et $t$ l'instant de son 
acquisition. On désigne par $I(x, y, t)$ l'intensité lumineuse instantanée de l'image nette ; ces deux intensités sont liées par la relation :

$u(x, y) \operatorname{Grad}(J(x, y, t))=I(x, y, 0)-I(x-a, y-b, 0)$ où :

$u(x, y)$ est la vitesse de déplacement du point de coordonnées $(x, y)$, supposée constante pendant la saisie de l'image.

$a$ et $b$ sont les coordonnées du vecteur de déplacement correspondant à la vitesse $u$ pendant le temps de pose.

On s'intéresse dans une première étape à la vitesse moyenne $v f$ du déplacement des pixels de l'image pendant le temps de pose. En Transformée de Fourier cette relation devient :

$$
2 \operatorname{i\pi q} v J(\vec{q}, t))=\operatorname{Sin}(2 \pi \vec{q} \vec{a}) I(\mathrm{q}, t)
$$

où $\mathrm{q}$ est un vecteur des fréquences spatiales.

On désigne maintenant par $A$ le déplacement moyen entre deux trames consécutives. Soient $I_{0}, I_{1}, I_{2}, I_{3}$, les intensités de quatre trames successives séparées de 1/50 s on peut alors écrire trois relations entre les Transformées de Fourier de ces trames du type :

$$
I_{1}(\vec{q})=\mathrm{e}^{-2 i \pi \vec{q} q A} I_{0}(\vec{q}) .
$$

En notant $J_{0}, J_{1}, J_{2}, J_{3}$, les intensités des images non traitées nous obtenons les relations :

$$
\begin{aligned}
& J_{3}(\vec{q})-J_{1}(\vec{q})=\mathrm{e}^{-2 i \pi \vec{q} q A}\left(J_{2}(\vec{q})-J_{0}(\vec{q})\right) \\
& J_{3}(\vec{q})-J_{2}(\vec{q})=\mathrm{e}^{-4 i \pi \vec{q} q A}\left(J_{1}(\vec{q})-J_{0}(\vec{q})\right) .
\end{aligned}
$$

Nous constatons que ces relations ne contiennent plus de termes dus au «flou de bougé ». On est donc ramené à estimer les fonctions de transfert entre les différences de deux couples de trames.

L'expression de cette estimation est :

$$
H(\vec{q})=\frac{X(\vec{q}) Y^{*}(\vec{q})}{|X(\vec{q})|^{2}+\beta^{2}}
$$

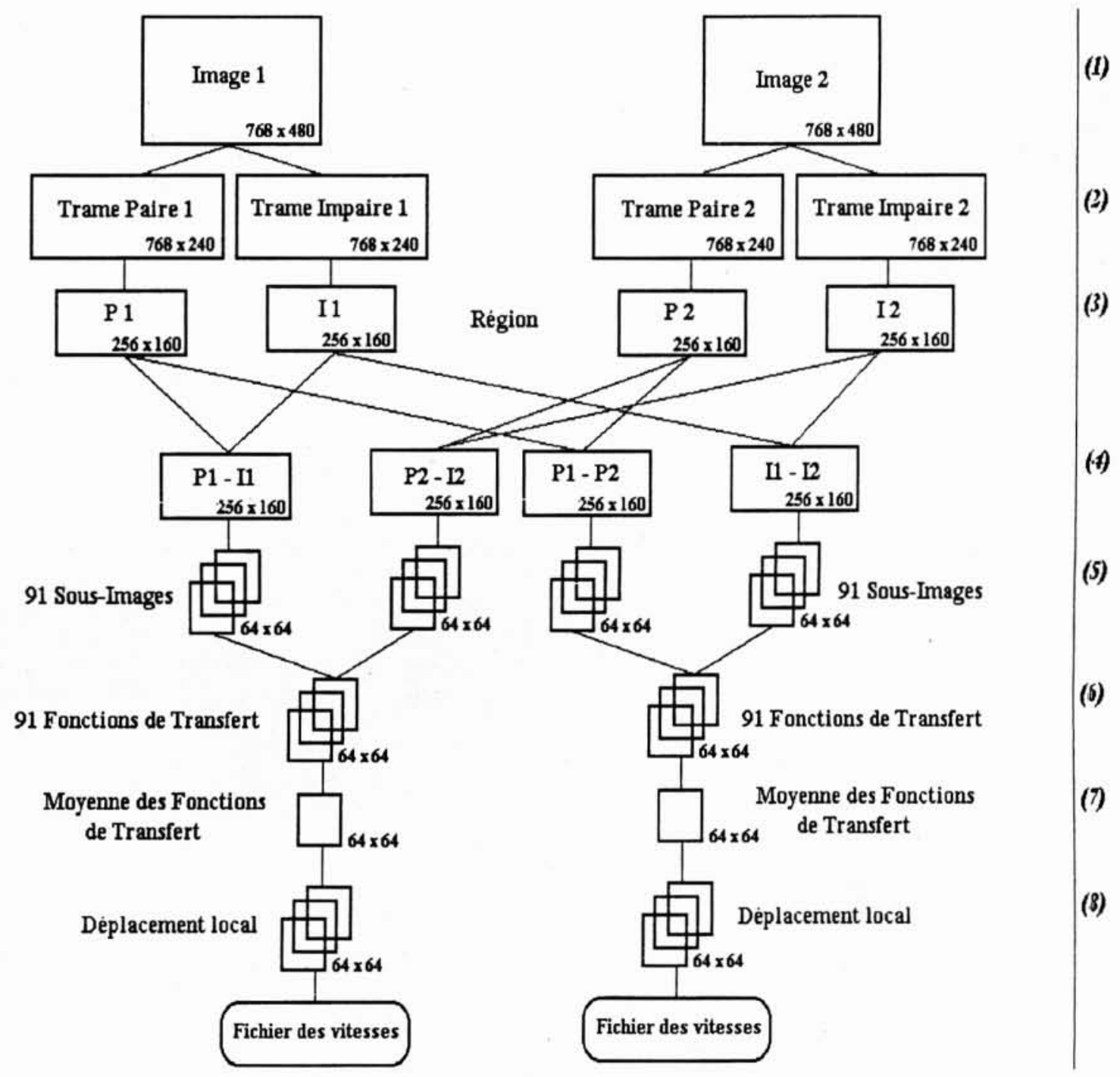

4. Synoptique de la méthode de traitement. 
$X(\vec{q})$ et $Y(\vec{q})$ représentent les différences des trames paires et impaires, $\beta$ est une estimation empirique de la densité spectrale du bruit. Pour diminuer l'influence du bruit, ce calcul a été réalisé sur un ensemble de sousimages de $64 \times 64$ pixels, ce qui diminue la résolution du déplacement mais améliore sa détection. La Transformée de Fourier Inverse $h(x, y)$ de la moyenne des fonctions $H(\vec{q})$ présente alors un pic très prononcé à l'extrémité du vecteur déplacement $A$.

Les valeurs des déplacements en fonction de la position dans l'image sont obtenues en recherchant dans chacune des sous-images le maximum de la fonction $h(x, y)$ au voisinage de l'extrémité du vecteur $A$. On obtient ainsi une première approche de la cartographie des vitesses à la surface de l'écoulement.

\subsection{Résultats obtenus}

Les films vidéo ont été pris par un camescope CCDV5000E (vidéo Hi8 PRO) de SONY utilisant le standard PAL. La numérisation des images a été effectuée par une carte Media Magic. Le traitement numérique a été réalisé à l'aide du logiciel DxImage de société DDS sur un calculateur HP 9000. Le synoptique de la méthode de traitement est indiqué dans la figure 4.

Les différentes étapes du calcul sont les suivantes :

(1) On utilise deux images consécutives, Image 1 et Image 2 ; Image 1 est représentée sur la figure 5.

(2) On extrait les deux trames de chacune des images.

(3) On travaille dans une région définie par le rectangle blanc $(256 \times 320)$ de la figure 5 .

(4) On travaille sur les deux couples de différences:

Trame Paire - Trame Impaire de l'image 1

Trame Paire - Trame Impaire de l'image 2

Le déplacement entre ces deux images est un vecteur $2 A$.

Trame Paire de l'image 1 - Trame Paire de l'image 2

Trame Impaire de l'image 1 - Trame Impaire de l'image 2.

Le déplacement entre ces deux images est un vecteur $A$.

(5) On extrait de chaque différence 91 sous-images $(64 \times 64)$ décalées de 16 pixels dans chacune des deux directions.

(6) On réalise les 91 estimations des fonctions $h(x, y)$ sur chaque différence.

(7) On détermine la position du maximum qui fournit le déplacement moyen dans la région étudiée sur chacune des moyennes.

(8) On recherche dans chacune des 91 fonctions $h(x, y)$ un maximum significatif au voisinage du déplacement moyen. On obtient ainsi une estimation du déplacement en chacun des 91 points de la région.

Ces déplacements sont représentés par des segments sur les figures 6 et 7.

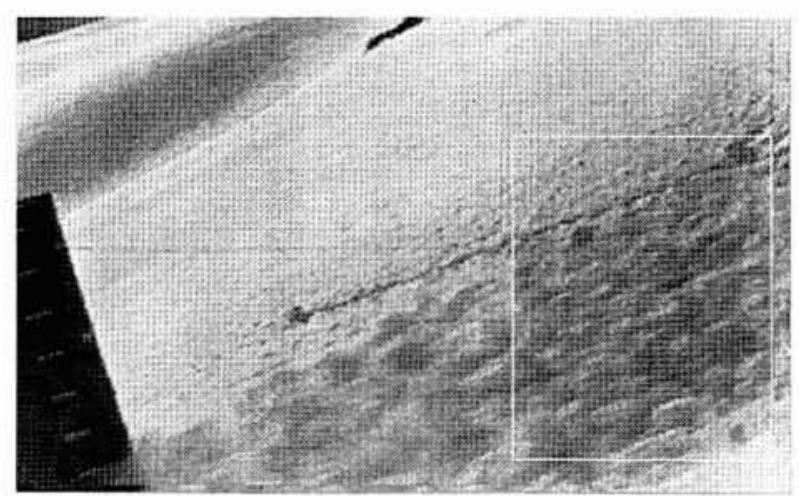

5. Image floue d'une avalanche. A gauche : un support de capteur profilé en aile d'avion. A droite : fenêtre déterminant la portion de surface étudiée.

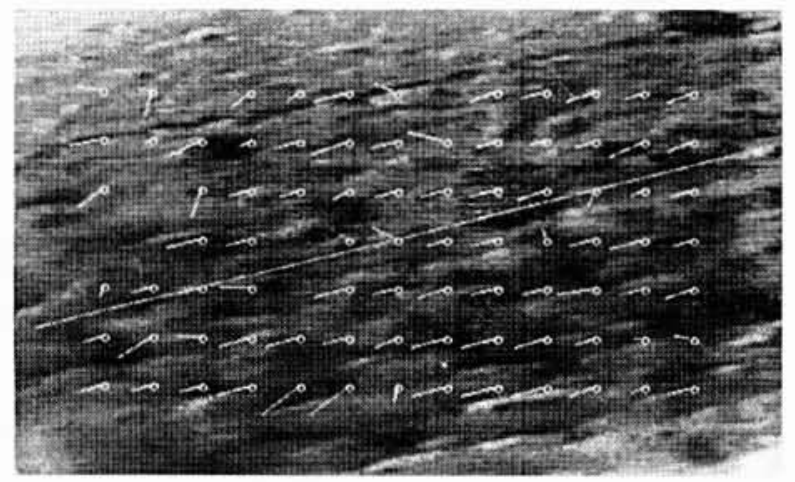

6. Déplacement du couple : Paire 1 - Paire 2 ; Impaire 1 Impaire 2.

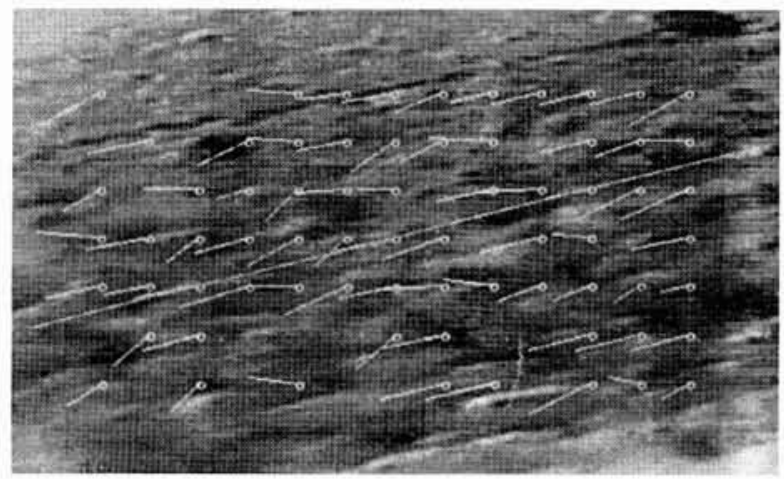

7. Déplacement du couple : Paire 1 - Impaire 1; Paire 2 - Impaire 2.

\subsection{Validation de ces résultats}

Les figures 8 et 9 représentent les moyennes des fonctions $h(x, y)$ obtenues dans chacun des deux traitements. On vérifie que le vecteur déplacement obtenu lorsque l'on travaille par différence de trames de deux images différentes, est $A(8,2)$. Le déplacement obtenu lorsque l'on travaille sur les différences de trames d'une même image est $A^{\prime}(16,4)$. Ceci est conforme aux hypothèses du modèle. Chaque fonction $h(x, y)$ présente, à quelques exceptions 

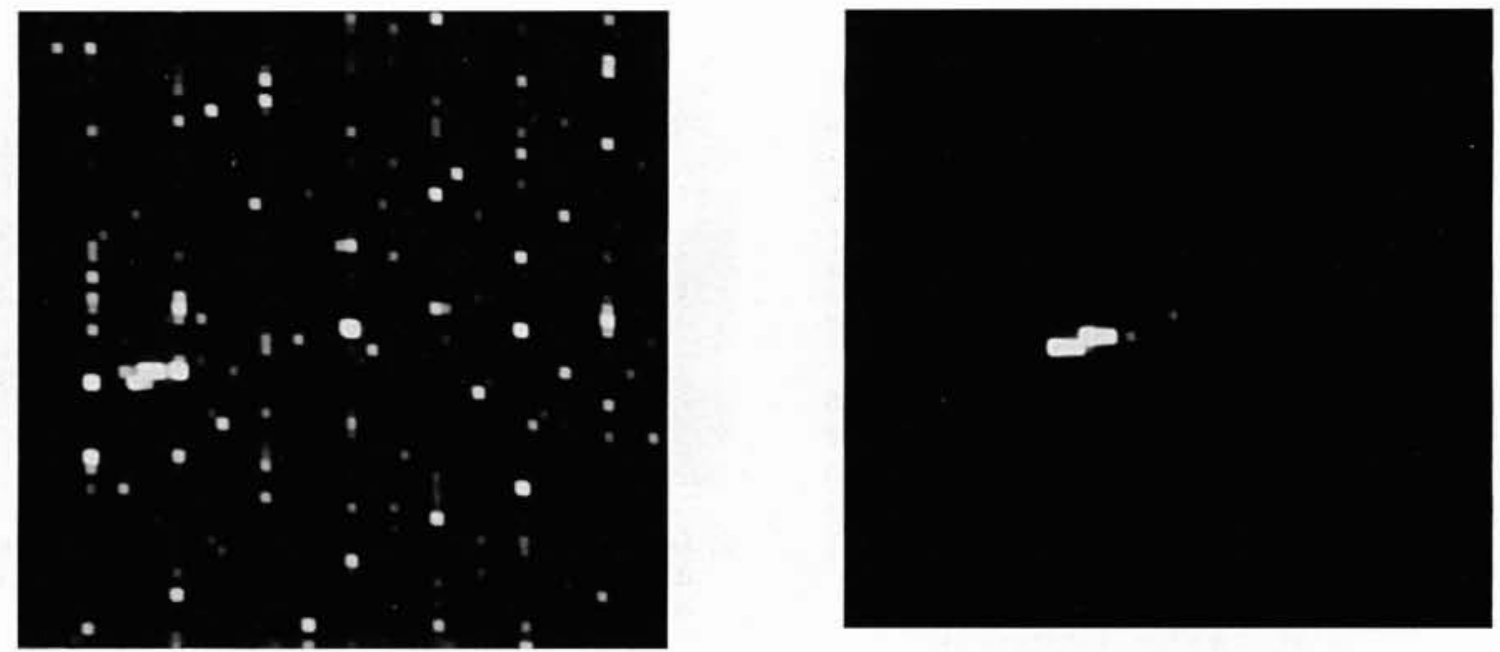

8 et 9. Moyenne des fonctions de transfert des couples : Paire 1-Impaire 1, Paire 2-Impaire 2, Paire 1-Paire 2, Impaire 1Impaire 2.

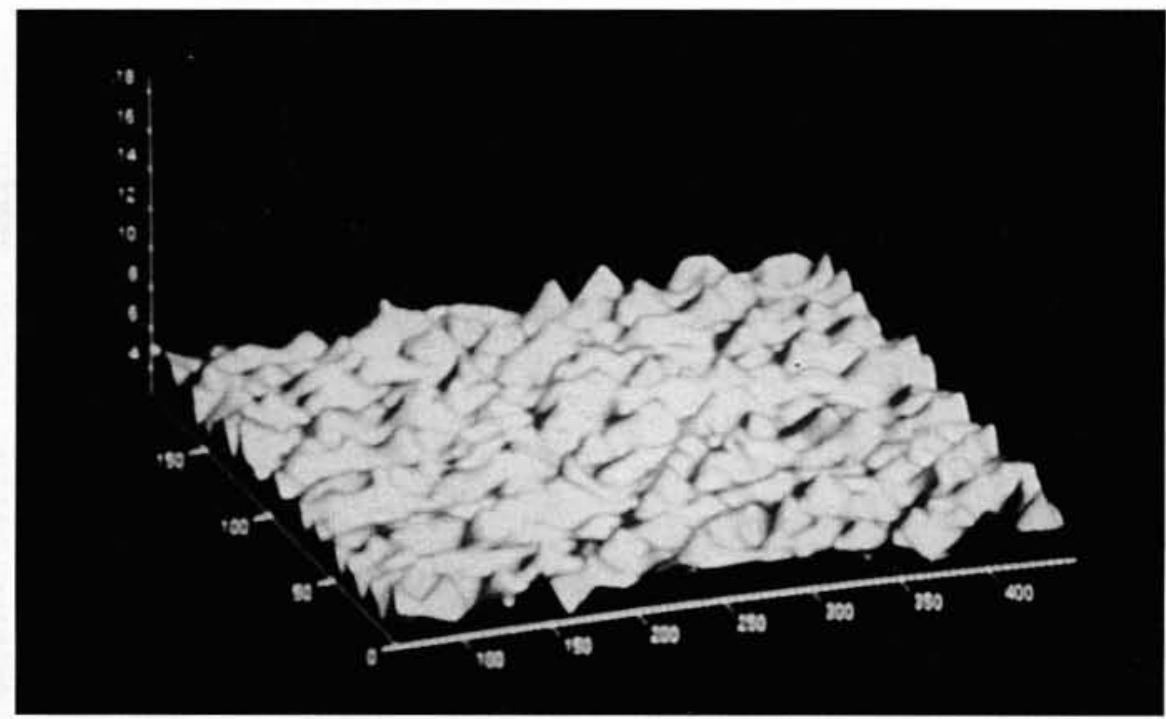

10. Vitesse $(\mathrm{m} / \mathrm{s})$ en fonction de la position (pixels). La position $(0,0)$ correspond au coin inférieur gauche des figures 6 et 7 .

près, un maximum dans une zone de plus ou moins 5 pixels autour du maximum moyen. Cela signifie que l'écoulement observé est assez uniforme, les fluctuations des déplacements n'excèdent pas $50 \%$ de la valeur moyenne. Pour illustrer les fluctuations de vitesses observées dans cette région de l'écoulement nous avons représenté sur la figure 10 , le module de la vitesse $\mathrm{m} / \mathrm{s}$ en fonction de la position (pixels).

D'autre part, un étalonnage de l'image réalisé dans le plan du support des capteurs situé à gauche de l'image sur la figure 5 donne une distance de 85 pixels par mètre, ce qui conduit à un déplacement moyen de $20 \mathrm{~cm}$ pendant $1 / 25 \mathrm{~s}$. La vitesse moyenne correspondante est donc de $5 \mathrm{~m} / \mathrm{s}$. Nous vérifions également sur les figures 6 et 7 que les déplacements mesurés décroissent en fonction de la distance à la caméra, ce qui s'explique par la profondeur de champ utilisée et donc par la différence entre la vitesse apparente sur le plan image et la vitesse réelle dans l'espace objet.

\section{CONCLUSIONS}

Ces premiers résultats prouvent qu'il est possible de mesurer par traitement d'images la distribution des vitesses à la surface d'un écoulement de neige dense en traitant numériquement des images obtenues dans des conditions standard de prise de vue. En l'état actuel de l'étude, une estimation correcte de la précision des résultats est encore difficile à fournir. Cependant, ces traitements effectués sur 


\section{IMAGERIE POUR LA CARTOGRAPHIE DES VITESSES}

un grand nombre de couples d'images ont toujours fourni des résultats cohérents. Plusieurs problèmes restent encore à traiter. L'étalonnage tridimensionnel des images en utilisant des repères connus sur le terrain permettra une évaluation exacte de la vitesse. L'évolution des vitesses au cours du temps nécessite un traitement systématique d'une grande série d'images qui fournira des données que l'on comparera aux modèles numériques appliqués au même site. Enfin l'étude de l'influence de la topographie du terrain sur la distribution des vitesses doit permettre une meilleure compréhension de la dynamique de l'écoulement.

Ce travail a été financé par le "Pôle Grenoblois d'Etudes et de Recherche pour la prévention des Risques Naturels " par le contrat de plan État-Région Rhône-Alpes, et par le programme européen "capital humain et mobilité".

\section{Références}

[1] MARCO O. (1994). - Instrumentation d'un site avalancheux. De l'utilisation des propriétés acoustiques de la neige et des techniques d'imagerie pour la mesure de paramètres physiques d'une avalanche dense. Thèse de doctorat de l'Université Joseph Fourier-Grenoble 1.

[2] Emery W.J., Thomas A.C., Colı.ins M.J. (1986). - An objective method for computing advective surface velocities from sequential infrared images, J. Geophys. Res., vol. 91 C11. pp. 12865-12878.

[3] Ninnis R.M., EMERY W.J. and Col.lins M.J. (1986). - An automated extraction of pack ice motion from Advanced Very High Resolution Radiometer imagery, J. Geophys. Res, vol. 91, no C9. pp. 10725-10734.
4] Wu Q.X., Pairman D., McNeill. S.J. and Barnes E.J. (1992). - Computing Advective Velocities from Satellite Images of Sea Surface Temperature, IEE Transactions on geoscience and remote sensing, vol. 30, no 1, pp. 166-175.

[5] LEESE J.A. and NOVAK C.S. (1971), - An automated technique for obtaining cloud motion from geosynchronous satellite data using cross correlation, J. Appl. Meteorol., vol. 10, pp. 118-132.

[6] GARCiA C.A.E. and Robinson S. (1989). - Sea surface velocities in shallow seas extracted from sequential coastal zone color scanner satellite data, J. Geophys. Res, vol. 94, no C9, pp. 12681-12691.

[7] GUBLER H. (1987), - Measurements an Modeling of snow Avalanche Speeds, IAHS Publication no 162, pp. 405-420, .

[8] Nunez J. and Llacer J. (Oct. 1993). - A General Bayesian Image Reconstruction Algorithm with Entropy Prior. Preliminary Application to HST Data, Publications of the Astronomical Society of the Pacific, vol. 105, pp. 1192-1208.

[9] GonZALEZ and WINTZ. - Digital Image Processing.

[10] ANDREwS H.C. and HUNT B.R. - Digital Image Restoring.

[11] WARD R.K. (1993). - Restoration of differently blurred versions of an image with measurement errors en the PSF's, IEEE Transactions on image processing, vol. 2, no 3 , pp. $369-381$

[12] BIEMOND J., LAGENDIJK R.L. and MERSEREAU R.M. (1990). - Iterative methods for image deblurring. Proceedings of the IEEE, vol. 78, no 5, pp. 856-883.

[13] BIEMOND J., VAN DER PUTTEN F.G. and WOODS J.W. (Apr. 1988). - Identification and restoration of images with symmetric noncausal blurs, IEEE Trans. Circ. Syst., vol. CAS-35, pp. 385-393. 